\title{
I-V and Switching Characteristics of Back Illuminated OPFET using Finite Difference Methods
}

\author{
Rajesh B. Lohani \\ Dept of Electronics and Telecommunication \\ Goa College of Engineering \\ Farmagudi, Ponda, Goa-India
}

\author{
Jaya V. Gaitonde \\ Dept of Electronics and Telecommunication \\ Goa College of Engineering \\ Farmagudi, Ponda, Goa-India
}

\begin{abstract}
OPFET (Optical Field Effect Transistor) is a useful device for optical communication and as photo detector. In this paper, the $\mathrm{I}-\mathrm{V}$ characteristics of the back illuminated OPFET are plotted using finite difference methods by solving the without time dependent continuity equations in which the incident radiation is allowed to enter through the substrate by inserting a fiber partially into the substrate. The switching time of the device has also been plotted.
\end{abstract}

\section{General Terms \\ Performance, Theory.}

\section{Keywords}

OPFET, photo detector, finite difference methods, back

illumination.

\section{INTRODUCTION}

At present, optical fiber communication plays an important role in cable communication technology for wideband, multimedia and high-speed applications [1]. The fundamental physical mechanism behind optical illumination is the absorption of photons in the valence band of the material thereby creating electrons and holes. The photo-generated electrons contribute to the drain-source current when a drain-source voltage is applied and the holes develop a photo-voltage at the Schottky junction and the p-n junction of the device resulting in the modulation of the channel conductance [2].

A continuity equation is a differential equation that describes the transport of some kind of conserved quantity; in this case, it is the carrier density. Solving the continuity equation to obtain the current-voltage characteristics is a major area of research. Existing researches include perturbation method [3] and analytical methods [4]-[7] to solve the continuity equation of the OPFET. In this paper, the finite difference methods are used to solve the continuity equations using finite difference equations to approximate derivatives.

\section{THEORY}

The schematic structure of the ion-implanted GaAs OPFET with back illumination is shown in Fig.1, having the fiber inserted partially into the substrate so that the absorption takes place in both substrate and active region. The drain-source current flows along the $\mathrm{x}$-direction and the illumination is incident along the $y$-direction of the device. Electron-hole pairs are generated due to absorption of photons in the neutral substrate region, the
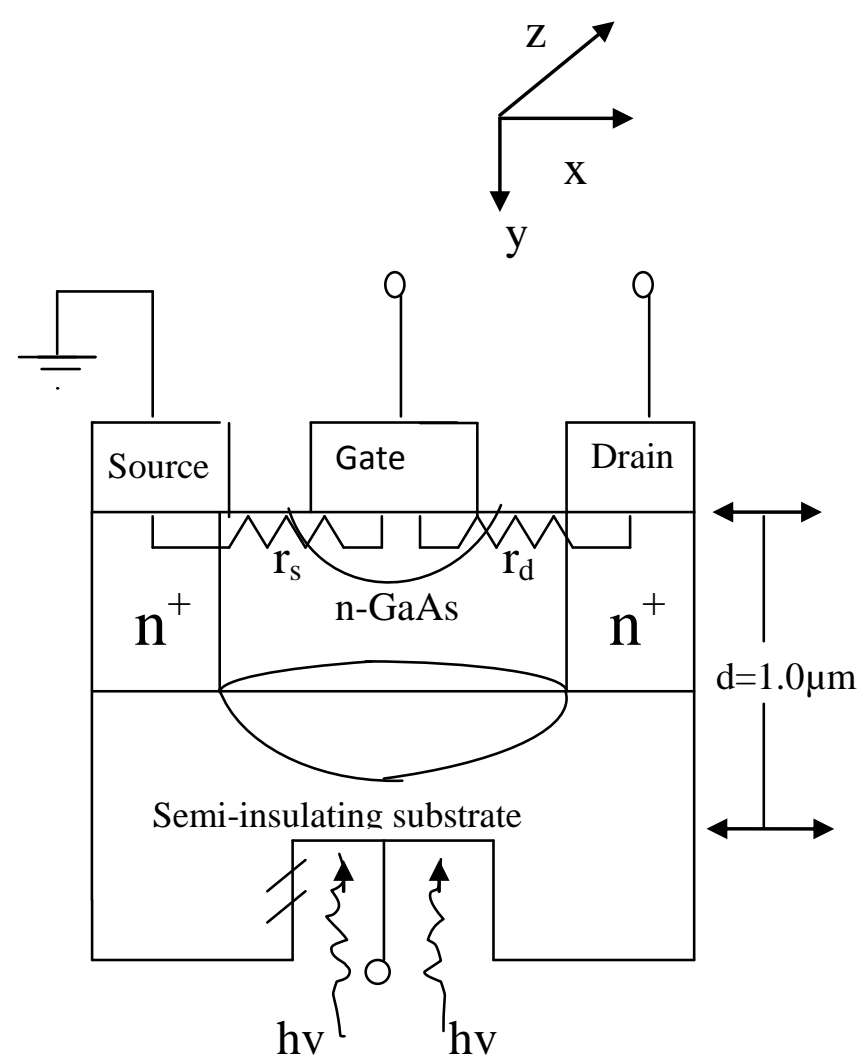

Fig 1: Schematic structure of the device with fiber inserted partially into the substrate [2].

active layer-substrate depletion region, the neutral channel region and the Schottky junction depletion region. The optically generated electrons move toward the channel and contribute to the drain-source current when a drain-source voltage is applied while the holes move in the opposite direction. When these holes cross the junction a photovoltage is developed. This voltage being forward biased reduces the depletion width of both the junctions [2].

To use a finite difference method to solve a problem, one must first discretize the problem's domain. This is done by dividing the domain into a finite number of equally spaced points as shown in figure 2 [8]. 

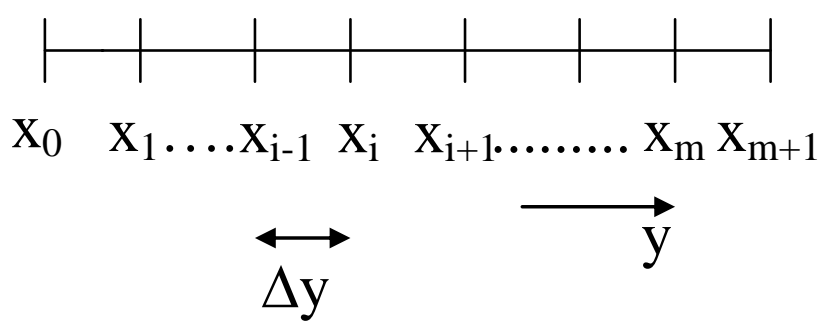

Fig 2: Finite difference discretization of the problem domain along the $y$ direction with equally spaced points or nodes [8].

The derivative $\partial \mathrm{x} / \partial \mathrm{y}$ can be approximated at the point $\mathrm{x}_{\mathrm{i}}$ in several ways. The forward differencing technique uses

$$
\frac{\partial x}{\partial y}=\frac{x_{i+1}-x_{i}}{\Delta y}
$$

The backward differencing technique uses

$$
\frac{\partial x}{\partial y}=\frac{x_{i}-x_{i-1}}{\Delta y}
$$

The central differencing technique uses

$$
\frac{\partial x}{\partial y}=\frac{x_{i+1}-x_{i-1}}{2 \Delta y}
$$

Approximations of differential equations of order 2 can be given as:

$$
\frac{\partial^{2} x}{\partial y^{2}}=\frac{x_{i+1}-2 x_{i}+x_{i-1}}{\Delta y^{2}}
$$

The time dependent continuity equation for electrons can be represented in one dimension as [2]

$$
\frac{\partial n(y, t)}{\partial t}=\frac{1}{q} \frac{\partial J_{n}(y, t)}{\partial y}+G-\frac{n(y, t)}{\tau_{n}}-\frac{R_{s} \tau_{n}}{S_{n}}
$$

The time dependent continuity equation for holes can be represented in one dimension as [2]

$$
\frac{\partial p(y, t)}{\partial t}=-\frac{1}{q} \frac{\partial J_{p}(y, t)}{\partial y}+G-\frac{p(y, t)}{\tau_{p}}-\frac{R_{s} \tau_{p}}{S_{p}}
$$

$\mathrm{G}$ is the volume generation rate of carriers and is assumed to vary exponentially with distance. Taking the surface as the reference since the radiation is incident from the surface side, follows the following relation [2]:

$$
G=\alpha \phi e^{-\alpha(d-y)}
$$

where $d$ is the surface to substrate thickness, $\alpha$ is the absorption coefficient per unit length and $\Phi$ is the radiation flux density per unit area per second.
$\mathrm{J}_{\mathrm{n}}$ is the electron current density given by [2]

$$
J_{n}=q v_{y} n+q D_{n} \frac{\partial n}{\partial y}
$$

$\mathrm{J}_{\mathrm{p}}$ is the hole current density given by [2]

$$
J_{p}=q v_{y} p-q D_{p} \frac{\partial p}{\partial y}
$$

In the above equations, $\tau_{\mathrm{n}}$ is the electron lifetime, $\mathrm{n}$ is the number of electrons, $p$ is the number of holes, $R_{s}$ is the surface recombination rate, $S_{n}$ is the surface recombination velocity for electrons and $S_{p}$ is the surface recombination velocity for holes. $\mathrm{q}$ is the electronic charge, $\mathrm{v}_{\mathrm{y}}$ is the carrier velocity in the $\mathrm{y}$ direction, $D_{n}$ is the diffusion constant for electrons and $D_{p}$ is the diffusion constant for holes.

The term $\mathrm{R}_{\mathrm{s}}$ is given by [2], [9]

$$
R_{s}=\frac{N_{T} k_{n} k_{p}\left(n_{s} p_{s}-n_{t} p_{t}\right)}{k_{n}\left(n_{s}+n_{t}\right)+k_{p}\left(p_{s}+p_{t}\right)}
$$

where $\mathrm{n}_{\mathrm{s}}=\alpha \Phi \tau_{\mathrm{n}}$ and $\mathrm{p}_{\mathrm{s}}=\alpha \Phi \tau_{\mathrm{p}} . \mathrm{N}_{\mathrm{T}}$ is the trap density, $\mathrm{k}_{\mathrm{p}}$ is the capture factor for holes, $k_{n}$ is the capture factor for electrons, $n_{s}$ and $\mathrm{p}_{\mathrm{s}}$ are the surface carrier concentrations for electrons and holes respectively, which take the values $n_{t}$ and $p_{t}$ respectively when the Fermi level lies in the traps. $\tau_{\mathrm{p}}$ is the hole lifetime.

The incident radiation is assumed to be modulated by a signal of frequency $\omega$. Thus under small signal condition [2]

$$
\phi=\phi_{0}+\phi_{1} e^{j \omega t}
$$

where "zero" indicates the dc value and "one" indicates the ac value.

There are two effects induced by the optical illumination in the device. These are the photoconductive effect and photovoltaic effect. The absorption of photon energy within the n-type layer and the semi-insulating substrate generates excess carriers and increases the conductivity of these regions, thus setting up a photoconductive current in addition to any drain current. This process arise from the separation and collection of generated electron-hole pairs along the longitudinal direction of the channel, and therefore have their strongest dependence on drain to source voltage rather than on gate to source voltage. This is the photoconductive effect.

The photovoltaic processes arise from the collection of photogenerated carriers into the space charge regions. Thus there are two different kinds of photovoltaic effects: the internal photovoltaic effect and the external photovoltaic effect. Since a difference in doping levels exists between the n-type layer and the semi-insulating substrate a potential barrier, or depletion region, is set up at their interface as occurs with p-n junctions. When photon absorption occurs at this interface barrier, the generated carriers then diffuse and are swept further by the junction electric field, the electrons toward the active channel and the holes toward the substrate. A photovoltage is developed. This junction is forward bias which opposes the interface barrier potential causing a decrease in the channel-substrate depletion 
region width resulting in a greater opening of the conducting channel. This is the internal photovoltaic effect.

The depletion region set up by an external reverse bias at the gate of the device provides an additional area for photon absorption. When illuminated, this region acts in an almost identical manner to that of a Schottky photodiode. The high internal electric field associated with the Schottky gate junction caused electron-hole pairs generated by the photon absorption to separate. In addition, electron-hole pairs generated by photon absorption away from the depletion region (within the n-type epitaxial layer) can contribute to the photo effect by diffusing into the edge of the depletion region before recombination takes place. This is the external photovoltaic effect.

When the photon energy is equal to, or greater than, the bandgap of the semiconductor, the photo-excitation of free carriers (electron-hole pairs) can take place.

In the neutral region, the transport mechanism of carriers is due to diffusion and recombination. So the continuity equation is represented by a second order differential equation.

The frequency dependent ac equation is given by [2]

$$
\frac{\partial^{2} n}{\partial y^{2}}-\frac{n}{D_{n} \tau_{\omega n}}=-\frac{\alpha \phi_{1} e^{-\alpha(d-y)}}{D_{n}}
$$

First we consider neutral channel region. In this paper, we are not considering time so the component $\mathrm{e}^{\mathrm{j} \omega \mathrm{t}}$ in equation (11) is not there. Also, we are doing ac analysis. So, only $\Phi_{1}$ component is there. The effect of surface recombination is not present in case of electrons since only the presence of negative traps has been assumed at or close to the surface.

For $\partial^{2} n / \partial y^{2}$, we substitute equation (4) and we get

$$
\frac{\partial^{2} n}{\partial y^{2}}=\frac{n_{i+1}-2 n_{i}+n_{i-1}}{\Delta y^{2}}
$$

where " $i$ ", " $i+1$ " and " $\mathrm{i}-1$ " indicate the position and $\Delta y$ is the space step.

The term $\mathrm{e}^{-\alpha(\mathrm{d}-\mathrm{y})} \mathrm{using}$ finite difference method can be written as

$$
e^{-\alpha(d-y)}=e^{-\alpha d}\left(e^{\alpha y+\alpha \Delta y / 2}-e^{\alpha y-\alpha \Delta y / 2}\right)
$$

where $\Delta y$ is the space step.

Substituting the finite difference approximation for the second order partial derivative and for the term $\mathrm{e}^{-\alpha(\mathrm{d}-\mathrm{y})}$ in equation (12), the following equation is obtained:

$$
\begin{gathered}
\frac{n_{i+1}-2 n_{i}+n_{i-1}}{\Delta y^{2}}-\frac{n_{i}}{D_{n} \tau_{\omega n_{i}}}= \\
-\frac{\alpha \phi_{1} e^{-\alpha d}\left(e^{\alpha y_{i}+\alpha \Delta y / 2}-e^{\alpha y_{i}-\alpha \Delta y / 2}\right)}{D_{n}}
\end{gathered}
$$

The above equation can be written as

$$
\begin{aligned}
& D_{n} \tau_{\omega n_{i}}\left[n_{i+1}-2 n_{i}+n_{i-1}\right]-\Delta y^{2}\left[n_{i}\right]= \\
& -\alpha \phi_{1} e^{-\alpha d} \Delta y^{2} \tau_{\omega n_{i}}\left(e^{\alpha y_{i}+\alpha \Delta y / 2}-e^{\alpha y_{i}-\alpha \Delta y / 2}\right)
\end{aligned}
$$

For $\mathrm{m}$ number of finite difference points, $\mathrm{i}=2$ to $\mathrm{m}-1$ is substituted in equation (16), so that $\mathrm{m}-2$ equations with $\mathrm{m}$ unknowns are obtained. So the boundary conditions are used to know 2 out of the $m$ unknowns. The boundary conditions are

At $\mathrm{y}=\mathrm{y}_{\mathrm{dg} 1}$,

$n=\alpha \phi_{1} \tau_{\omega n} e^{-\alpha\left(d-y_{d g 1}\right)}$

and at $\mathrm{y}=\mathrm{y}_{\mathrm{ds} 1}$,

$n=\alpha \phi_{1} \tau_{\omega n} e^{-\alpha\left(d-y_{d s 1}\right)}$

where $d$ is the surface to substrate thickness, $\tau_{\oplus n}$ is the lifetime of the electrons under ac condition, $\mathrm{y}_{\mathrm{dg} 1}$ is the extension of the Schottky junction depletion region in the channel measured from the surface and $\mathrm{y}_{\mathrm{ds} 1}$ is the extension of the $\mathrm{p}$-n junction depletion region in the channel measured from the surface.

$\tau_{\omega n}$ is given by [2]

$$
\frac{1}{\tau_{\omega n}}=\frac{1}{\tau_{n}}+j \omega
$$

where $\tau_{\mathrm{n}}$ is the lifetime of the electrons and $\omega$ is the frequency.

$\mathrm{y}_{\mathrm{dg} 1}$ is given as [2]

$$
y_{d g 1}=\left[\frac{2 \varepsilon}{q N_{d r}}\left(\phi_{B}-\Delta+v(x)-v_{g s}-V_{O P 1}\right]^{\frac{1}{2}}\right.
$$

assuming the abrupt junction approximation where $\mathrm{N}_{\mathrm{dr}}$ is the equivalent constant doping concentration, $\Phi_{\mathrm{B}}$ is the Schottky barrier height, $\Delta$ is the position of Fermi level below the conduction band, $\mathrm{v}(\mathrm{x})$ is the voltage drop beneath the gate which varies from 0 at the source end and $\mathrm{V}_{\mathrm{ds}}$ at the drain end. $\mathrm{V}_{\mathrm{ds}}$ is the drain to source voltage and $\mathrm{v}_{\mathrm{gg}}$ is the gate to source voltage. $\mathrm{V}_{\mathrm{OP} 1}$ is the photovoltage across the Schottky junction.

$\mathrm{y}_{\mathrm{ds} 1}$ is given as [2]

$$
y_{d s 1}=a-\frac{N_{A}}{N_{d r}}\left[\frac{2 \varepsilon}{q N_{A}}\left(v_{b i}+v(x)-v_{b s}-V_{O P 2}\right)\right]^{\frac{1}{2}}
$$

assuming abrupt junction approximation where $\mathrm{N}_{\mathrm{A}}$ is the substrate doping concentration, $\mathrm{v}_{\mathrm{bi}}$ is the built-in potential, $\mathrm{v}_{\mathrm{bs}}$ is the substrate potential and $\mathrm{V}_{\mathrm{OP} 2}$ is the photovoltage across the channel-substrate junction. 
$\mathrm{V}_{\mathrm{OP} 1}$ and $\mathrm{V}_{\mathrm{OP} 2}$ are calculated as follows:

The external photovoltage $\mathrm{V}_{\mathrm{OP} 1}$ across the Schottky junction is calculated using the relation [2]

$$
V_{O P 1}=\frac{k T}{q} \ln \left(\frac{J_{p}}{J_{s}}\right)=\frac{k T}{q} \ln \left(\frac{q v_{y} p(0)}{J_{s 1}}\right)
$$

where $J_{s 1}$ is the reverse saturation current density across the Schottky junction. $\mathrm{p}(0)$ is the number of holes crossing the junction at $\mathrm{y}=0 . \mathrm{k}$ is the Boltzmann constant, $\mathrm{T}$ is the absolute temperature and $\mathrm{q}$ is the electronic charge.

The internal photovoltage $\mathrm{V}_{\mathrm{OP} 2}$ across the channel-substrate junction is obtained using the relation [2]

$$
V_{O P 2}=\frac{k T}{q} \ln \left(\frac{J_{p}(a)}{J_{s 2}}\right)=\frac{k T}{q} \ln \left(\frac{q v_{y} p(a)}{J_{s 2}}\right)
$$

where $J_{\mathrm{s} 2}$ is the reverse saturation current density for the $p-n$ junction. $p(a)$ is the number of holes crossing the junction at $\mathrm{y}=\mathrm{a}, \mathrm{a}$ is the active layer thickness. $\mathrm{p}(0)$ and $\mathrm{p}(\mathrm{a})$ is obtained by solving the continuity equation for holes in the Schottky junction depletion region and the channel-substrate depletion region respectively.

Now by substituting the boundary conditions, m-2 equations with $\mathrm{m}-2$ unknowns are obtained.

These equations are solved by writing the coefficients of the unknowns in a matrix. It will be a $(\mathrm{m}-2) \mathrm{x}(\mathrm{m}-2)$ matrix. This matrix will be multiplied by the $(\mathrm{m}-2) \mathrm{x} 1$ matrix of the unknowns $\mathrm{n}_{2}$ to $\mathrm{n}_{\mathrm{m}-2}$. This will be the left hand side of the equation. The right hand side will be the $(\mathrm{m}-2) \mathrm{x} 1$ matrix consisting of the values obtained from the remaining terms. Now using matrix inversion we will get the values of $n$. Then the boundary values are appended to the values $\mathrm{n}_{2}$ to $\mathrm{n}_{\mathrm{m}-2}$. This is done for all the boundaries obtained by taking each value of $y_{\mathrm{dg} 1}$ and $\mathrm{y}_{\mathrm{ds} 1}$.

Now, these values of $\mathrm{n}$ are used to calculate the amount of charge in the neutral channel region which is the integration or summation of number of electrons in each space step multiplied by the electronic charge. It is given as

$$
Q_{c h}=q\left[n_{1} \Delta y+n_{2} \Delta y+n_{3} \Delta y+\ldots+n_{m} \Delta y\right]
$$

This is done for each pair of boundaries.

The current in the neutral channel region is given by [2]

$$
I_{c h}=\frac{\mu Z}{L} \int_{0}^{V_{d s}} Q_{c h} d v
$$

where $V_{d s}$ is the drain to source voltage, $\mu$ is the mobility of electrons, $\mathrm{Z}$ is the channel width and $\mathrm{L}$ is the channel length. Here the limits of integration are: 0 as the lower limit and $V_{d s}$ as the upper limit. We use summation to calculate the current since the discrete equivalent of integration is summation. The limits 0 and $\mathrm{V}_{\mathrm{ds}}$ is the voltage $\mathrm{v}(\mathrm{x})$ beneath the gate which varies as 0 at the source end and $V_{d s}$ at the drain end. For $m$ finite difference points there will be $\mathrm{m}$ values of the voltage $\mathrm{v}(\mathrm{x})$. The value obtained by taking the difference between any two consecutive values is $\mathrm{dv}$. This is the voltage drop beneath the gate as we go from source to drain. Then we start from the source end and calculate the value of charge between the first pair of boundaries from equation (20). Then we multiply this value of charge with the first voltage drop at the source end. Then we calculate the value of charge between the second pair of boundaries from equation (20). Then we multiply this value of charge with the second voltage drop. Similarly we repeat this procedure for the other pair of boundaries till we reach the $\mathrm{m}^{\text {th }}$ pair of boundaries. Then we add all the values and multiply the resulting value with $\mu \mathrm{Z} / \mathrm{L}$ to obtain the value of channel current for the corresponding value of $\mathrm{V}_{\mathrm{ds}}$. Since we vary $\mathrm{V}_{\mathrm{ds}}$ between 0 to $\mathrm{V}$ volts taking $\mathrm{m}$ number of points, the value of channel current is calculated for each value of $\mathrm{V}_{\mathrm{ds}}$. So, we get $\mathrm{m}$ values of channel current for $\mathrm{m}$ values of $\mathrm{V}_{\mathrm{d} s}$.

Now we consider the neutral substrate region.

The boundary conditions are

At $\mathrm{y}=\mathrm{y}_{\mathrm{w} 1}$,

$n=\alpha \phi_{1} \tau_{\omega n} e^{-\alpha\left(d-y_{w 1}\right)}$

and at $\mathrm{y}=\mathrm{d}$,

$n=\alpha \phi_{1} \tau_{\omega n}$

$\mathrm{y}_{\mathrm{w} 1}$ is given as [2]

$y_{w 1}=y_{d s 1}+\left[\frac{2 \varepsilon}{q N}\left(v_{b i}+V_{d s}-v_{b s}\right)\right]^{\frac{1}{2}}$

where

$\frac{1}{N}=\frac{1}{N_{A}}+\frac{1}{N_{d r}}$

Now the equation (16) is solved using the boundary conditions given by equations (26) and (27) in the similar manner as described for the neutral channel region.

Now the amount of charge in the neutral substrate region is calculated as

$Q_{s u b}=q\left[n_{1} \Delta y+n_{2} \Delta y+n_{3} \Delta y+\ldots+n_{m} \Delta y\right]$

This is done for each pair of boundaries.

The current in the neutral substrate region is given by [2] 


$$
I_{s u b}=\frac{\mu Z}{L} \int_{0}^{V_{d s}} Q_{s u b} d v
$$

In the depletion region, the transport mechanism of carriers is due to drift and recombination. So the continuity equation is represented by a first order differential equation.

The frequency dependent ac equation is given by [2]

$$
\frac{\partial n}{\partial y}-\frac{n}{v_{y} \tau_{\omega n}}+\frac{\alpha \phi_{1} e^{-\alpha(d-y)}}{v_{y}}=0
$$

First we consider the Schottky junction depletion region.

For $\partial \mathrm{n} / \partial \mathrm{y}$ we substitute equation (3) and we get

$$
\frac{\partial n}{\partial y}=\frac{n_{i+1}-n_{i-1}}{2 \Delta y}
$$

where " $\mathrm{i}+1$ " and " $\mathrm{i}-1$ " indicate the position and $\Delta \mathrm{y}$ is the space step.

Substituting the finite difference approximation for the partial derivative and for the term $\mathrm{e}^{-\alpha(\mathrm{d}-\mathrm{y})}$ in equation (32), the following equation is obtained:

$$
\begin{aligned}
& {\left[\frac{n_{i+1}-n_{i-1}}{2 \Delta y}\right]-\frac{n_{i}}{v_{y} \tau_{\omega n_{i}}}} \\
& +\frac{\alpha \phi_{1} e^{-\alpha d}}{v_{y}}\left(e^{\alpha y_{i}+\alpha \Delta y / 2}-e^{\alpha y_{i}-\alpha \Delta y / 2}\right)=0
\end{aligned}
$$

The above equation can be written as

$$
\begin{aligned}
& v_{y} \tau_{\omega n_{i}}\left[n_{i+1}-n_{i-1}\right]-2 \Delta y\left[n_{i}\right] \\
& +\alpha \phi_{1} e^{-\alpha d} \tau_{\omega n_{i}} 2 \Delta y\left(e^{\alpha y_{i}+\alpha \Delta y / 2}-e^{\alpha y_{i}-\alpha \Delta y / 2}\right)=0
\end{aligned}
$$

The boundary conditions are

At $\mathrm{y}=0$,

$$
n=\alpha \phi_{1} \tau_{\omega n} e^{-\alpha d}
$$

and at $\mathrm{y}=\mathrm{y}_{\mathrm{dg} 1}$,

$$
n=\alpha \phi_{1} \tau_{\omega n} e^{-\alpha\left(d-y_{d g 1}\right)}
$$

where $\mathrm{y}_{\mathrm{dg} 1}$ is given by equation (20).

Now the equation (35) is solved using the boundary conditions given by equations (36) and (37) in the similar manner as described for the neutral channel region.
Now the amount of charge in the Schottky junction depletion region is calculated as

$$
Q_{\text {dep } 1}=q\left[n_{1} \Delta y+n_{2} \Delta y+n_{3} \Delta y+\ldots+n_{m} \Delta y\right]
$$

This is done for each pair of boundaries.

The current in the Schottky junction depletion region is given by [2]

$$
I_{d e p 1}=\frac{\mu Z}{L} \int_{0}^{V_{d s}} Q_{d e p 1} d v
$$

Now we consider the active layer-substrate depletion region.

The boundary conditions are

$$
\begin{aligned}
& \text { At } \mathrm{y}=\mathrm{y}_{\mathrm{ds} 1}, \\
& n=\alpha \phi_{1} \tau_{\omega n} e^{-\alpha\left(d-y_{d s 1}\right)}
\end{aligned}
$$

and at $\mathrm{y}=\mathrm{y}_{\mathrm{w} 1}$,

$$
n=\alpha \phi_{1} \tau_{\omega n} e^{-\alpha\left(d-y_{w 1}\right)}
$$

where $\mathrm{y}_{\mathrm{ds} 1}$ is given by equation (21) and $\mathrm{y}_{\mathrm{w} 1}$ is given by equation (28).

Now the equation (35) is solved using the boundary conditions given by equations (40) and (41) in the similar manner as described for the neutral channel region.

Now the amount of charge in the active layer-substrate depletion region is calculated as

$$
Q_{\text {dep } 2}=q\left[n_{1} \Delta y+n_{2} \Delta y+n_{3} \Delta y+\ldots+n_{m} \Delta y\right]
$$

This is done for each pair of boundaries.

The current in the active layer-substrate depletion region is given by [2]

$$
I_{d e p 2}=\frac{\mu Z}{L} \int_{0}^{V_{d s}} Q_{d e p 2} d v
$$

The back illuminated device consists of a p-type uniformly doped semi-insulating substrate followed by an epitaxially grown ion-implanted active layer of n-type doping. The ion implanted profile is represented by the Gaussian distribution given by [2], [10]

$$
N(y)=\frac{Q}{\sigma \sqrt{2 \pi}} \exp \left[-\left(\frac{y-R_{p}}{\sigma \sqrt{2}}\right)^{2}\right]
$$

where $\mathrm{Q}$ is the implanted dose, $\sigma$ is the straggle parameter and $R_{p}$ is the projected range. 
Table I. Values of different parameters used for calculation $[2$

\begin{tabular}{|l|l|l|l|}
\hline Parameter & Name & Value & Unit \\
\hline$\sigma$ & Straggle Parameter & $0.383 \times 10^{-7}$ & $(\mathrm{~m})$ \\
\hline $\mathrm{R}_{\mathrm{p}}$ & Projected Range & $0.861 \times 10^{-7}$ & $(\mathrm{~m})$ \\
\hline$\mu_{\mathrm{n}}$ & Electron mobility & 0.45 & $\left(\mathrm{~m}^{2} / \mathrm{V} . \mathrm{s}\right)$ \\
\hline $\mathrm{Z}$ & Channel Width & $100 \times 10^{-6}$ & $(\mathrm{~m})$ \\
\hline$\alpha$ & $\begin{array}{l}\text { Absorption } \\
\text { Coefficient }\end{array}$ & $1.0 \times 10^{6}$ & $\left(\mathrm{~m}^{-1}\right)$ \\
\hline$\tau_{\mathrm{n}}$ & Electron Lifetime & $1.0 \times 10^{-6}$ & $(\mathrm{~s})$ \\
\hline$\tau_{\mathrm{p}}$ & Hole Lifetime & $1.0 \times 10^{-8}$ & $(\mathrm{~s})$ \\
\hline $\mathrm{v}_{\mathrm{y}}$ & $\begin{array}{l}\text { Carrier Velocity in } \\
\text { ydirection }\end{array}$ & $1.2 \times 10^{5}$ & $(\mathrm{~m} / \mathrm{s})$ \\
\hline $\mathrm{d}$ & $\begin{array}{l}\text { Thickness of the } \\
\text { device including } \\
\text { substrate }\end{array}$ & $1.0 \times 10^{-6}$ & $(\mathrm{~m})$ \\
\hline $\mathrm{L}$ & Channel Length & $3.0 \times 10^{-6}$ & $(\mathrm{~m})$ \\
\hline $\mathrm{a}$ & $\begin{array}{l}\text { Active Layer } \\
\text { Thickness }\end{array}$ & $0.25 \times 10^{-6}$ & $(\mathrm{~m})$ \\
\hline$\Delta$ & $\begin{array}{l}\text { Position of Fermi } \\
\text { Level below the } \\
\text { conduction band }\end{array}$ & 0.02 & $(\mathrm{eV})$ \\
\hline$\Phi_{\mathrm{B}}$ & $\begin{array}{l}\text { Schottky Barrier } \\
\text { Height }\end{array}$ & 0.9 & $(\mathrm{eV})$ \\
\hline $\mathrm{N}_{\mathrm{T}}$ & Trap Density & $1.0 \times 10^{15}$ & $\left(\mathrm{~m}^{-2}\right)$ \\
\hline $\mathrm{k}_{\mathrm{p}}$ & $\begin{array}{l}\text { Capture factor for } \\
\text { holes }\end{array}$ & $3.1 \times 10^{-17}$ & $(\mathrm{~m} / \mathrm{s})$ \\
\hline $\mathrm{k}_{\mathrm{n}}$ & $\begin{array}{l}\text { Capture factor for } \\
\text { electrons }\end{array}$ & $3.1 \times 10^{-15}$ & $\left(\mathrm{~m}^{3} / \mathrm{s}\right)$ \\
\hline $\mathrm{v}_{\mathrm{bs}}$ & Substrate potential \\
\hline $\mathrm{N}_{\mathrm{A}}$ & $\begin{array}{l}\text { Substrate doping } \\
\text { concentration }\end{array}$ & 0.0 & $(\mathrm{~V})$ \\
\hline $\mathrm{N}_{\mathrm{dr}}$ & $\begin{array}{l}\text { Equivalent constant } \\
\text { doping } \\
\text { concentration }\end{array}$ & $0.658 \times 10^{23}$ & $\left(\mathrm{~m}^{-3}\right)$ \\
\hline & & & \\
\hline & & & \\
\hline & & & \\
\hline & & & \\
\hline
\end{tabular}

The ac drain-source current due to ion-implantation is obtained using the relation [2]

$I_{\text {ion }}=\frac{\mu Z}{L} \int_{0}^{V_{d s}} Q_{i o n} d v$

where $\mathrm{Q}_{\text {ion }}$ is the ac channel charge due to ion-implantation and is calculated from [2]

$$
Q_{\text {ion }}=q \int_{y_{d g 1}}^{y_{d s 1}} N(y) d y
$$

$\mathrm{Z}$ is the channel width and $\mathrm{L}$ is the channel length.

The ac component of the total drain-source current is contributed by the carriers due to ion-implantation and optical generation in the channel and substrate regions. It can be represented as [2]

$I_{d s}($ total $)=I_{i o n}+I_{c h}+I_{d e p}+I_{s u b}$ where $I_{d e p}$ is the sum of currents $I_{d e p 1}$ and $I_{d e p 2}$.

The switching time of the back illuminated OPFET depends on the active channel thickness. The switching time is computed for different active layer thickness expressed by the following equation [11]

$$
\tau=\frac{\left[L\left(\phi_{B}-V_{O P 1}-v_{g s}\right)^{1 / 2}\right]}{\left[V_{s}\left[\left(\frac{q N_{d a v g} a^{2}}{2 \varepsilon}\right)^{1 / 2}-\left(\phi_{B}-V_{O P 1}-v_{g s}\right)^{1 / 2}\right]\right]}
$$

where $\mathrm{q}$ is the electronic charge, $\mathrm{L}$ is the channel length, $\mathrm{N}_{\text {davg }}$ is the average channel doping concentration, $a$ is the active layer thickness, $v_{\mathrm{gs}}$ is the gate to source voltage, $\Phi_{\mathrm{B}}$ is the Schottky barrier height, $\mathrm{V}_{\mathrm{OP} 1}$ is the photovoltage across the Schottky junction, $\mathrm{V}_{\mathrm{s}}$ is the saturation velocity.

Table 1 shows the values of different parameters used in calculation.

\section{RESULTS AND DISCUSSIONS}

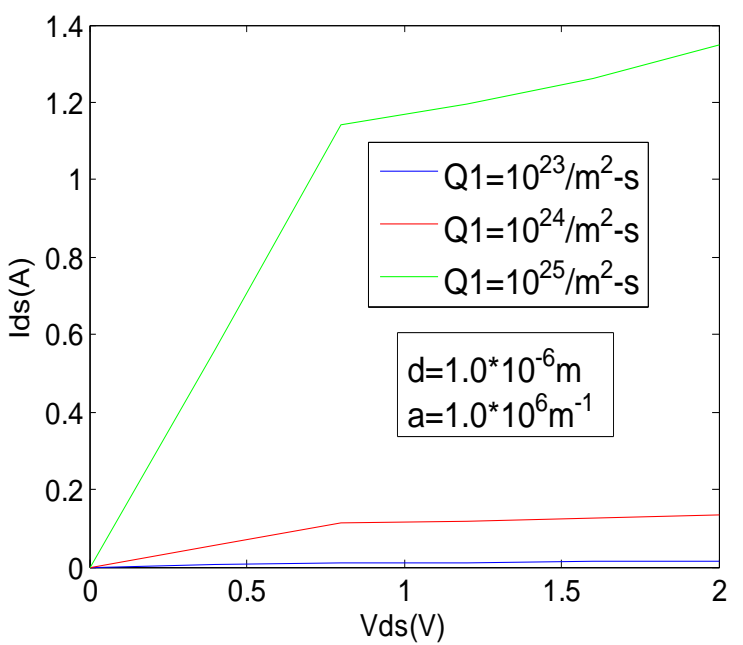

Fig 3: Ids-Vds characteristics for different radiation flux densities.

Figure 3 shows the current-voltage characteristics of the back illuminated device for different radiation flux densities. It is seen that with the increase in the radiation flux density, the current increases. This is because with the increase in the radiation flux density, the volume generation rate of carriers increases. That is why more carriers are generated and hence the current increases. There are several mechanisms by which the drain current increases due to illumination which explains the photo effects in the device. Incident photons cause a change in the built-in voltage of the gate junction and by this mechanism, the drain current increases. Both the photoconductivity effect in the source-gate and the drain-gate regions and the change of the gate depletion width are responsible for the increase of the drain current. With illumination, the potential barrier is lowered and the position of the depletion edge on the channel side of the 
channel/substrate interface moves toward the interface. The resulting effective channel width, therefore, increases. This increases the drain current. Another reason for the increase of the drain current is the reduction of the source to gate and gate to drain resistances in series with the active channel due to illumination [12].

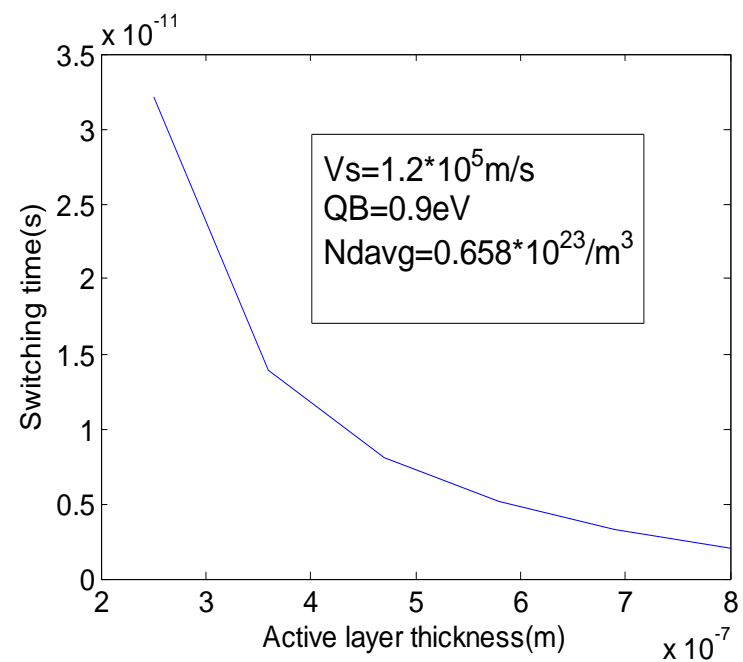

Fig 4: Switching time v/s active layer thickness.

Figure 4 shows the plot of switching time versus active layer thickness of the back illuminated device. It is seen that with the increase in active layer thickness, the switching time decreases.

\section{CONCLUSION}

The continuity equation for electrons has been solved using finite difference methods to plot the I-V characteristics of the back illuminated device for different radiation flux densities. Since the current increases with the increase in the radiation flux density the device with back illumination can be used for the design of high speed optical detector in communication by increasing the radiation flux density and for the design of optical switch by increasing the active layer thickness. The device can be used as a detector since with small change in the radiation flux density, there is large change in the drain to source current. The device can be used as a high speed optical detector because of the high current obtained due to reduction in the width of both the depletion regions due to photovoltaic effect and the increase in the conductivity of the channel and substrate regions due to photoconductive effect. This is because the response speed of the detector is determined by the width of the depletion region. Lesser the width, higher the speed. Also, the switching time decreases with the increase in the active layer thickness and the value of switching time is low. So, the device can be used as a switch.

\section{ACKNOWLEDGEMENT}

Authors thanks Dr. B.B. Pal, ITBHU and Dr. R. P. R. C. Aiyar, IIT, Mumbai for providing constant help and encouragement and necessary guidance.

\section{REFERENCES}

[1] K. Balasubadra, A. Arulmary, V. Rajamani, K. Sankaranarayanan, "Two Dimensional Numerical Modeling and Simulation of a Uniformly doped GaAs MESFET Photodetector", Journal of Optical Communications, 29(2008)4, pp. 194-201.

[2] Nandita Saha Roy and B. B. Pal, "Frequency-Dependent OPFET Characteristics with Improved Absorption under Back Illumination", Journal of Lightwave Technology, Vol. 18, No. 4, pp. 604-613, April 2000.

[3] Youssef Zebda and S. Abu_Helweh, "AC Characteristics of Optically Controlled MESFET (OPFET)", Journal of Lightwave Technology, Vol. 15, No. 7, pp. 1205-1212, July 1997.

[4] Nandita Saha Roy, B. B. Pal, and R. U. Khan, "Analysis of GaAs OPFET with Improved Optical Absorption under Back Illumination", IEEE Transactions on Electron Devices, Vol. 46, No. 12, pp. 2350-2353, December 1999.

[5] Nandita Saha Roy, B. B. Pal, and R. U. Khan, "FrequencyDependent Characteristics of an Ion-Implanted GaAs MESFET with Opaque Gate Under Illumination", Journal of Lightwave Technology, Vol. 18, No. 2, pp. 221-229, February 2000.

[6] M. K. Verma and B. B. Pal, "Analysis of Buried Gate MESFET Under Dark and Illumination", IEEE Transactions on Electron Devices, Vol. 48, No. 9, pp. 2138-2142, September 2001.

[7] B. B. Pal, Shubha, K. Honey Kumar and R. U. Khan, "Frequency Dependent Behaviour of an Ion Implanted GaAs OPFET considering the Photovoltaic Effect and Gate Depletion Width Modulation", Solid State Electronics, Vol.38, No. 5, pp. 1097-1102, 1995.

[8] Jaya V. Gaitonde and Rajesh B. Lohani, "One Dimensional Finite Difference Simulation of Back Illuminated OPFET", In Proceedings of International Conference \& Workshop on Emerging Trends in Technology 2011, Mumbai, VolumeII, pp. 1258-1261, February 25th \& 26th 2011.

[9] Sunita Mishra, V. K. Singh and B. B. Pal, "Effect of Radiation and Surface Recombination on the Characteristics of an Ion-Implanted GaAs MESFET", IEEE Transactions on Electron Devices, Vol. 37, No. 1, pp. 2-10, January 1990.

[10] Shubha R. Saxena, R. B. Lohani, R. U. Khan, B. B. Pal, "Generalized dc model of GaAs optical field effect transistor considering ion-implanted profile", Optical Engineering, 37(04), April 1998, pp.1343-1352.

[11] S. N. Chattopadhyay, N. Motoyama, A. Rudra, A. Sharma, S. Sriram, C. B. Overton, and P. Pandey, "Optically Controlled Silicon MESFET Modeling Considering Diffusion Process", Journal of Semiconductor Technology and Science, Vol. 7, No. 3, pp. 196-208, September 2007.

[12] Shih-Hsien Lo, and Chien-Ping Lee, "Numerical Analysis of the Photoeffects in GaAs MESFETs", IEEE Transactions on Electron Devices, vol. 39. no. 7, pp. 15641570, July 1992. 